\title{
DIAGNÓSTICO DO PLANEJAMENTO DE PEQUENOS E MÉDIOS PRODUTORES DE PinUs taeda DOS ESTADOS DO PARANÁ E SANTA CATARINA
}

\author{
DIAGNOSIS FOR PLANNING OF SMALL AND MEDIUM SIZE FOREST FARMERS OF \\ Pinus taeda ON PARANÁ AND SANTA CATARINA STATES, BRAZIL
}

\author{
Kauê Augusto Oliveira Nascimento', Renato César Gonçalves Robert², Nelson Yoshihiro Nakajima ${ }^{3}$ \\ Rafael Oliveira Brown ${ }^{4}$ \\ 1, 2, 3,4 Universidade Federal do Paraná, Curitiba, Paraná, Brasil-kaue.nascimento@ufpr.br, \\ renatorobert@hotmail.com,nelson.nakajima@ufpr.br\&brownrafael@gmail.com
}

\begin{abstract}
RESUMO
A importância econômica da produção do gênero Pinus é notória como base de sustentação de uma economia setorial que possui significância para a economia nacional. O objetivo deste trabalho foi investigar os agentes e os fatores que determinaram os regimes de manejo florestal de Pinus taeda de pequenos e médios produtores das mesorregiões Norte Catarinense e Metropolitana de Curitiba. Foram aplicados questionários semiestruturados e perguntas abertas consequentes de auditorias para certificação florestal, com perguntas relacionadas ao manejo florestal aplicado e cadeia produtiva, totalizando 29 propriedades nos estados do Paraná e Santa Catarina. Detectou-se três contextos diferentes no desenvolvimento da cadeia produtiva e 2 regimes de manejo diferentes: 1o regime (18 anos de ciclo) e 20 regime (15 anos). A demanda industrial local e o nível de conhecimento da atividade foram apontados pelos produtores como os mais influentes para tomada de decisão quanto aos regimes de manejo e envolvimento na atividade, explicando a maior preferência pelo $2 \circ$ regime de manejo (62\%), que realiza menos intervenções na floresta durante o ciclo. A inserção de novas tecnologias na indústria de beneficiamento trouxe mudanças na demanda dos sortimentos, causando a decisão de redução do tempo de rotação de Pinus taeda para uso múltiplo para 15 anos, por pequenos e médios produtores da região.
\end{abstract}

PALAVRAS-CHAVE: Cadeia produtiva, Manejo florestal, Mercado de madeira.

\section{ABSTRACT}

The economic importance of production of the genus Pinus is notorious as a basis for sustaining a sectoral economy that has significance for national economy. The goal of this research was to investigate the agents and factors that determined the forest management regimes of Pinus taeda of small and medium producers of mesoregions Northern Catarinense and Metropolitan of Curitiba. Semi-structured questionnaires and interviews resulting from audits for forest certification were applied, with questions related to forest management and supply chain, totaling 29 properties in the states of Paraná and Santa Catarina. Three different cases were detected in the development of the supply chain and two different management approaches: first model ( 18 years rotation) and second model ( 15 years rotation). The level of knowledge of the activity and manufacturing demands were pointed out by the producers as decision-maker factors regarding management regimes and involvement in the activity, explaining the greater preference for the 2 nd management approach (62\%), which generates fewer interventions in the forest stands during rotation. The insertion of new technologies in the manufacturing industry brought changes in assortments demand, causing the decision to reduce the rotation of Pinus taeda to 15 years cycle, by small and medium producers of the region.

KEYWORDS: Supply chain, Forest management, Timber market. 


\section{INTRODUÇÃO}

Os gêneros Pinus e Eucalyptus formam a maior parte dos plantios no Sul do Brasil e principal origem de produtos florestais nessa região (KOHLER et al., 2015). A produtividade de um plantio florestal está relacionada com as práticas de manejo aplicadas, com os objetivos de agregar valor, melhorar suas características e obter multiprodutos (KOHLER et al., 2015). Um dos desafios em empreendimentos florestais é o emprego adequado de técnicas de manejo, visando o aumento da produtividade sem que isso aumente demasiadamente os custos envolvidos (DAVID et al., 2017). Dentre essas técnicas destaca-se o desbaste, que leva em consideração quando (período em anos), como (critério de seleção das árvores) e quanto (número de árvores por hectare ou área basal que deve permanecer) (SILVA et al., 2005).

Além de questões técnicas, a produtividade florestal leva em consideração questões do mercado como a taxa de atratividade, oferta, demanda e preço da madeira, nas quais todas essas variáveis participam das tomadas de decisão (JONES et al., 2010). Em seu estudo, Vasques et al. (2007) enfatizam a importância econômica do gênero Pinus para a economia, afirmando que em termos de números e cifras, é notória sua importância como base de sustentação de uma economia setorial que possui significância para a economia nacional. Os autores concluíram que a movimentação significativa de recursos relacionados a dimensão do setor produtivo de madeira roliça, a cadeia produtiva da madeira e as demandas agregadas são vitais para a região e para o país.

Dessa forma, a necessidade de compreensão dos padrões de produção e dos fatores que determinam as tomadas de decisão dos pequenos e médios produtores, quanto ao manejo florestal aplicado, são determinantes para o planejamento ao longo prazo da cadeia produtiva, justificando assim, a realização deste trabalho. Dessa forma, este estudo teve o objetivo de investigar os agentes e os fatores que determinam os regimes manejo florestal de Pinus taeda L. na região, de forma a gerar informações para o diagnóstico da cadeia produtiva regional.

\section{MATERIAL E MÉTODOS}

Foram aplicados, entre os meses de maio e julho de 2018, questionários semiestruturados e perguntas abertas a 29 pequenos e médios produtores florestais das mesorregiões Metropolitana de Curitiba, Norte Catarinense, Serrana e Oeste Catarinense. O questionário utilizado foi fruto das auditorias realizadas para validação de origem da madeira de uma grande empresa da região, visando ao selo de certificação de controle de origem da madeira.

O questionário e as perguntas foram amplos e relacionados aos agentes envolvidos na cadeia produtiva (equipe própria, terceiros, revendedores), às características da floresta, às técnicas empregadas de manejo florestal, às finalidades de produção, à estrutura física e às máquinas utilizadas nas operações de silvicultura e colheita, dentre outras informações pertinentes à contextualização regional, presentes na Tabela 1.

Tabela 1. Temas abordados e tópicos questionados aos produtores.

\begin{tabular}{|c|c|}
\hline Temas & Tópicos questionados \\
\hline $\begin{array}{l}\text { Manejo } \\
\text { florestal } \\
\text { aplicado }\end{array}$ & $\begin{array}{l}\text { - Características da floresta (espaçamento, } \\
\text { idade e área plantada) } \\
\text { - Tratos silviculturais e desbastes (intensidade e } \\
\text { tipo) }\end{array}$ \\
\hline $\begin{array}{c}\text { Cadeia } \\
\text { produtiva }\end{array}$ & $\begin{array}{l}\text { - Agentes envolvidos na colheita (equipe } \\
\text { própria ou terceiros) } \\
\text { - Compradores da madeira (indústria ou } \\
\text { intermediário) } \\
\text { - Motivações para atuar no setor }\end{array}$ \\
\hline $\begin{array}{c}\text { Mão de obra } \\
\text { utilizada }\end{array}$ & $\begin{array}{l}\text { - Número de trabalhadores e tipo (próprios ou } \\
\text { terceiros) } \\
\text { - Qualificação dos trabalhadores }\end{array}$ \\
\hline $\begin{array}{l}\text { Maquinários e } \\
\text { ferramentas }\end{array}$ & $\begin{array}{l}\text { - Equipamentos utilizados na silvicultura, poda e } \\
\text { colheita } \\
\text { - Estado de conservação e funcionamento dos } \\
\text { equipamentos } \\
\text { - Posse dos maquinários (próprio ou terceiros) }\end{array}$ \\
\hline $\begin{array}{l}\text { Segurança e } \\
\text { saúde do } \\
\text { trabalho }\end{array}$ & $\begin{array}{l}\text { - Presença e estado dos EPIs necessários } \\
\text { - Estrutura física necessária (áreas de vivência, } \\
\text { alimentação, hidratação, entre outros), } \\
\text { segundo as premissas da certificação florestal }\end{array}$ \\
\hline
\end{tabular}

Além das questões pré-estabelecidas, foram feitas perguntas abertas para coleta de quaisquer informações relevantes à análise dos dados. O esforço amostral, bem como a localização das propriedades por mesorregião estão listados abaixo (Tabela 2).

Os produtores foram classificados em dois tipos: pequenos e médios produtores. Os pequenos produtores foram caracterizados por pequenos proprietários de terra, como chácaras, sítios e pequenas fazendas de uso múltiplo, em que o plantio florestal é somente uma das fontes de renda, geralmente associada à criação de animais e agricultura de pequena escala. Estes produtores veem a produção florestal como uma poupança ou renda extra. 
Tabela 2. Localização e número de propriedades envolvidas neste estudo.

\begin{tabular}{cccc}
\hline UF & Mesorregiões & Municípios & No \\
\hline PR & $\begin{array}{c}\text { Metropolitana } \\
\text { de Curitiba }\end{array}$ & $\begin{array}{c}\text { Agudos do Sul, Campo do } \\
\text { Tenente, Piên, Rio Negro, } \\
\text { Quitandinha, Tijucas do Sul. }\end{array}$ & 10 \\
SC & $\begin{array}{c}\text { Oeste } \\
\text { Catarinense }\end{array}$ & Lebon Régis. & 01 \\
SC & $\begin{array}{c}\text { Campo Alegre, Corupá, } \\
\text { Catarinense } \\
\text { Itaiópolis, Mafra, Monte } \\
\text { Castelo, Papanduva, Rio } \\
\text { Negrinho, Santa Terezinha, } \\
\text { São Bento do Sul, Schroeder, } \\
\text { Timbó Grande. }\end{array}$ & 17 \\
SC & Serrana & $\begin{array}{c}\text { Campo Belo do Sul, Santa } \\
\text { Cecília. }\end{array}$ & 01 \\
\hline & \multicolumn{3}{c}{ Total } \\
\hline
\end{tabular}

Na Figura 1 é apresentada a disposição espacial das fazendas.

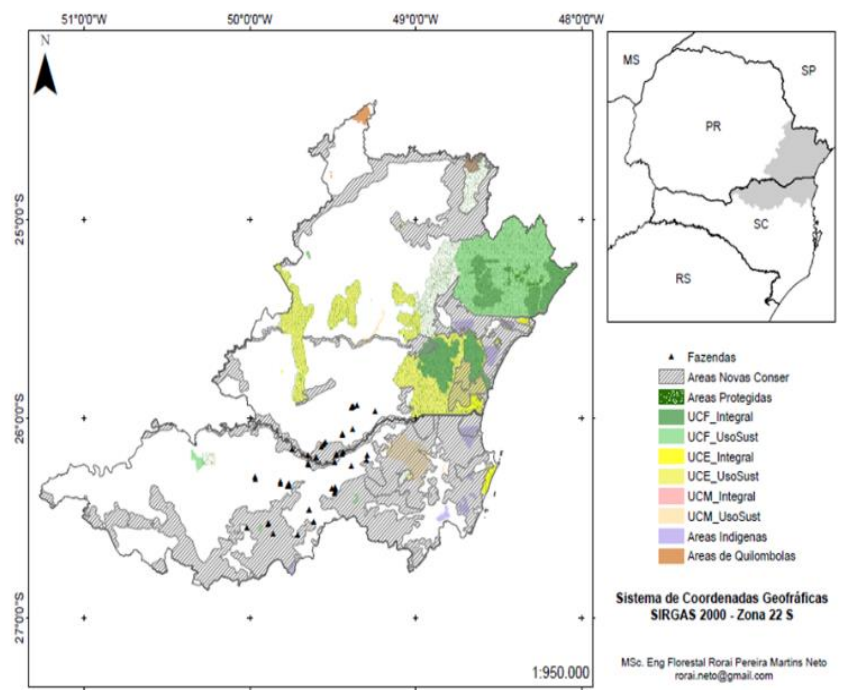

Figura 1. Disposição espacial das fazendas avaliadas (pontos pretos) sob o contexto de uso do solo nas mesorregiões contempladas.

Os pequenos produtores detêm pouco domínio técnico sobre a atividade e investem pouco em equipamentos e tratos silviculturais, bem como no manejo adequado da área. Estes produtores tendem a priorizar a maximização do volume de madeira e ciclos mais curtos, em detrimento do planejamento florestal para sortimentos maiores com maior retorno econômico. Estes vendem a floresta em pé para um empreiteiro, que se torna dono da madeira e realiza a colheita e o transporte da matéria prima para o destino.

Os médios produtores são proprietários de áreas entre 64 e 240 hectares (4 a 15 módulos fiscais) geralmente voltadas exclusivamente para a produção florestal, como forma de investimento. Estes produtores conhecem bem o mercado madeireiro e possuem bom conhecimento técnico da atividade, investindo em equipamentos e pessoal para manutenção da floresta. Estes negociam a madeira diretamente com a indústria de beneficiamento, de acordo com a finalidade de uso para cada sortimento. A colheita é realizada por equipes próprias ou por terceiros contratados pelo produtor (empreiteiros), dependendo do caso. O transporte é realizado por terceiros contratados. Nota-se que o produtor contrata os terceiros, permanecendo como o dono da matéria-prima retirada e negociando-a diretamente com as empresas interessadas na manufatura.

\section{RESULTADOS E DISCUSSÃO}

\section{Agentes da cadeia produtiva}

Foram encontrados no estudo três tipos diferentes de agentes atuantes: equipes próprias, empreiteiros e revendedores. As equipes próprias constituíram-se de funcionários diretos dos produtores florestais, com carteira assinada, contratados para a manutenção e colheita das florestas. Neste caso, os maquinários e todos os equipamentos eram de propriedade do produtor florestal.

Os empreiteiros constituíram-se de empresários prestadores de serviços que montaram equipes para colheita e transporte de madeira, tendo estabelecido com os produtores, contratos de compra e venda de madeira em pé, estabelecendo o período e quantidade de madeira a ser colhida, além de aspectos de qualidade nas operações. Estes possuíram maquinários e funcionários próprios, não tendo nenhuma relação trabalhista com o produtor além do contrato legal de prestação de serviços.

Os revendedores constituíram-se de prestadores de serviços exclusivos para o transporte florestal. Estes terceiros foram contratados pelos produtores ou empreiteiros somente para transportar a madeira ao destino. Entretanto, em alguns casos compraram a madeira nos sortimentos menores (menor valor de mercado) diretamente dos produtores florestais ou de terceiros, atuando de forma oportunista, devido ao desejo de limpeza da área e pouco interesse deles em sortimentos mais finos, tendo atuado assim como revendedor da madeira colhida.

\section{Contextualização da cadeia produtiva}

No diagnóstico foram detectados três casos frequentes 
de desenvolvimento da cadeia produtiva. No primeiro caso, os pequenos produtores vendem as toras para pequenas serrarias na região e os toretes (toras finas para processo) para cavaqueadoras de madeira próximos, que por sua vez, abastecem as grandes indústrias de painéis. No segundo caso, há mais um agente neste processo, o empreiteiro, que compra as árvores em pé de pequenos e médios produtores, realizam o corte e transporte das toras para serrarias e encaminham os toretes diretamente para a indústria ou são intermediados por empresas de cavaqueamento.

Na terceira situação, médios produtores que conhecem bem a atividade contratam uma empreiteira ou possuem equipes próprias, eles mesmos comercializam a madeira e geralmente já possuem uma lista definida de compradores para cada sortimento. Estes vendem para serrarias, laminadoras e cavaqueadoras de médio e grande porte. Alguns possuem indústria própria.

Um dos questionamentos referiu-se aos fatores de decisão que estimularam os produtores a trabalhar com a produção florestal. Diversos fatores foram apontados pelos produtores, dentre estes, a demanda contínua da indústria local, o nível de conhecimento técnico e de mercado da atividade foram determinantes, resultados demonstrados na tabela a seguir (Tabela 3).

Tabela 3. Fatores de decisão apontados pelos produtores para a escolha por empreendimentos florestais.

\begin{tabular}{cc}
\hline Fatores de decisão & \% Produtores \\
\hline Demanda da indústria & 48,3 \\
\hline Conhecimento técnico/mercado & 34,5 \\
\hline Liquidez da matéria prima & 13,8 \\
\hline Gosto pessoal pela atividade & 6,9 \\
\hline
\end{tabular}

Os valores ultrapassam $100 \%$, pois houve produtor que citou mais de um fator de decisão como relevante.

O tamanho da área de plantio demonstrou não ser influente nas escolhas de manejo dos produtores da região, a partir do momento que produtores pequenos que continham experiência e conhecimento do setor, atuavam ativamente na cadeia, produzindo, revendendo e trabalhando como terceiro para produtores maiores.

Abaixo, foram ilustradas as formas de organização da cadeia produtiva de madeira de Pinus taeda de pequenos e médios produtores nas regiões estudadas, conforme a Figura 2.
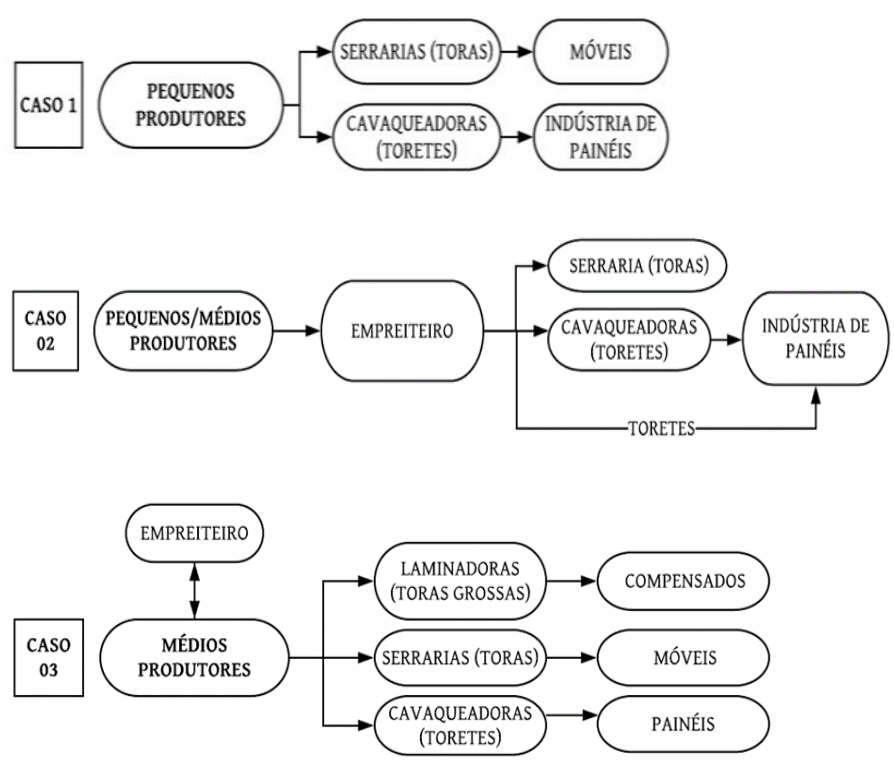

Figura 2. Casos encontrados de organização da cadeia produtiva de Pinus taeda dos pequenos e médios produtores nas mesorregiões estudadas.

\section{Regimes de manejo florestal}

Conforme a Tabela 4 a seguir, as propriedades utilizaram predominantemente o segundo regime de manejo, estas consistindo em todas as propriedades localizadas na mesorregião Metropolitana de Curitiba e em algumas da mesorregião Norte Catarinense, no caso, aquelas com maior facilidade de acesso às indústrias de painéis localizadas no estado do Paraná, sendo notável o efeito da demanda industrial no tipo de manejo. Os regimes de manejo foram agrupados em duas classes devido a padrões observados durante a aplicação dos questionários e perguntas realizadas nas auditorias de campo. Com pequenas variações, estas duas grandes classes representaram em sua totalidade os regimes de manejo florestal aplicados nas 29 propriedades estudadas.

As propriedades localizadas no Norte Catarinense utilizaram em sua maioria o primeiro regime, além de tratos silviculturais adicionais (poda baixa e alta). No primeiro regime, as fazendas localizadas no Norte Catarinense estão rodeadas por inúmeras serrarias, indústrias para produção de compensados e indústrias para confecção de móveis; demandando árvores de maior diâmetro com madeira de melhor qualidade e livres de nós, o que justifica a aplicação de podas. 
Tabela 4. Agrupamento dos regimes de manejo florestal aplicados pelos pequenos e médios produtores florestais.

\begin{tabular}{|c|c|c|c|}
\hline Regime de manejo & Finalidade & Espaçamento & Propriedades \\
\hline $\begin{array}{l}\text { - } 1 \text { o desbaste misto } \\
\text { aos } 9 \text { anos: retirada } \\
\text { da quinta linha e } \\
\text { 10\% das piores } \\
\text { árvores; } \\
\text { - Podas (até } 6 \mathrm{~m} \text { ). } \\
\text { - 20 desbaste seletivo } \\
\text { aos } 13-14 \text { anos: } \\
\text { retirada de } 50 \% \text { das } \\
\text { árvores. } \\
\text { - Corte raso: } 18 \text { anos }\end{array}$ & $\begin{array}{c}\text { Toras para } \\
\text { laminação } \\
\text { e serraria }\end{array}$ & $\begin{array}{c}2,5 \mathrm{~m} \times 2,5 \mathrm{~m} \\
\text { ou } \\
2,5 \mathrm{~m} \times 3,0 \mathrm{~m}\end{array}$ & $38 \%$ \\
\hline $\begin{array}{l}\text { 10 desbaste misto } \\
\text { aos } 12 \text { anos: } \\
\text { retirada da quarta } \\
\text { linha e } 25 \% \text { das } \\
\text { piores árvores. } \\
\text { - Corte raso: } 15 \text { anos. }\end{array}$ & $\begin{array}{l}\text { Toras para } \\
\text { serraria e } \\
\text { madeira } \\
\text { para } \\
\text { processo }\end{array}$ & $2,5 \mathrm{~m} \times 2,5 \mathrm{~m}$ & $62 \%$ \\
\hline
\end{tabular}

Além disso, devido à solidez da cadeia produtiva nessa região, muitos produtores conduzem os povoamentos desde o princípio com destinação a empresas específicas, seguindo os requisitos de qualidade e sortimento delas. Por outro lado, no contexto das áreas que aplicam o segundo regime, há a proximidade de duas grandes indústrias consumidoras de madeira de menores diâmetros para confecção de painéis, ou seja, árvores mais finas, sem necessidade de poda. Dessa forma, pode-se afirmar que estes efeitos ocorreram devido à demanda das indústrias existentes em cada mesorregião, ou seja, as indústrias consumidoras nortearam as decisões técnicas de manejo florestal dos produtores.

Em seu estudo, Simioni \& Hoeflich (2007), com base na opinião de especialistas, identificaram os fatores críticos para a produção de madeira para energia em uma região próxima. Estes concluíram que a legislação ambiental e a certificação florestal foram variáveis em alta na prospecção da cadeia, fato esse que foi citado por diversos produtores entrevistados e considerados fatores limitantes, pelos altos custos e perda de área potencial produtiva.

Com a certificação da indústria visando ao mercado externo, a empresa precisou garantir que toda a madeira beneficiada tenha respeitado as premissas do selo de certificação adquirido para toda a cadeia de custódia. Dessa forma, os produtores tiveram que se adequar a uma série de variáveis para permanecerem elegíveis no fornecimento. Corroborando com essas perspectivas, Vasques et al. (2007) concluiu que para melhorar a eficiência da silvicultura e o manejo do gênero Pinus no Sul do Brasil, houve a necessidade de promoção de mecanismos para disseminação de técnicas que garantissem o processo embasado na sustentabilidade aplicada por todos os agentes, através de certificações e auditorias técnicas sistemáticas.

A demanda industrial, aliada ao conhecimento técnico da atividade, determinaram a maior preferência pelo segundo regime de manejo (62\% das fazendas), que estipulou menos intervenções na floresta e exigiu menor acompanhamento do plantio. Outro fator influente e citado pelos produtores mais ligados ao setor foi a inserção de tecnologias, relacionadas principalmente ao uso múltiplo da madeira nos diâmetros menores. Muitos desses produtores começaram a reduzir o ciclo do plantio, devido à nova possibilidade de laminação de toras nas classes intermediárias $(20-25 \mathrm{~cm}$ e $25-35 \mathrm{~cm}$ diâmetro ponta fina), a partir de tornos menores com maior aproveitamento na laminação.

Simioni \& Hoeflich (2007) apontaram fatores críticos para o desenvolvimento da indústria regional, verificando que a inserção de tecnologias e o planejamento estratégico regional foram os mais influentes, o que foi observado nos resultados encontrados neste presente estudo. Castro et al. (2008) afirmaram que a as relações de negociação entre os agentes da cadeia e a questão tecnológica foram considerados pontos cruciais para a análise da cadeia produtiva florestal, em um estudo diagnóstico no Rio Grande do Sul.

De acordo com as expectativas dos produtores, esperase com o passar dos anos que a rotação do Pinus taeda realizada por pequenos e médios produtores na região, estabilize-se por volta de 15 anos, devido ao maior volume médio de madeira com sortimento desejado, nessa faixa de idade. Esses resultados estão em consonância com o que é aplicado de forma geral no sul do Brasil. Segundo Moreira et al. (2017) as estimativas de estudos diagnósticos consideram ciclos de produção de 7 (sete) anos para o eucalipto e 15 anos para o gênero Pinus. Vale lembrar que a maioria dos produtores locais adquirem as mudas melhoradas da mesma empresa, que domina o comércio de mudas nas mesorregiões.

Esta intenção de redução da rotação foi expressa pelos produtores em áreas com plantios de mais de 20 anos de idade, onde estão aplicando corte raso para planejamento de um ciclo menor, visando volume por área em detrimento do volume individual. Com isso, pode-se esperar uma padronização cada vez maior dos regimes de manejo dos pequenos e médios produtores nas mesorregiões estudadas. Com a valorização atual das áreas produtivas, há algum tempo é observado o fomento ou arrendamento das pequenas e médias propriedades por 
grandes empresas do setor, visando a garantia de fornecimento próximo a indústria.

De acordo com Fischer \& Zylbersztajn (2012) os benefícios econômicos do fomento florestal se expressam na geração de rendas aos produtores participantes e contribuem na dinamização do desenvolvimento local, possibilitando o surgimento de novos arranjos produtivos. Os mesmos autores enfatizam que sob a ótica social, a renda gerada pelo fomento acarreta na fixação destes em suas terras, reduzindo, assim, as migrações para áreas urbanas.

\section{CONCLUSÕES}

A demanda comercial das empresas consumidoras de Pinus taeda da região norteiam o manejo florestal escolhido pelos pequenos e médios produtores. A inserção de novas tecnologias na indústria de beneficiamento é de conhecimentos dos produtores, que indicam uma redução da rotação dos plantios de Pinus taeda para 15 anos, nas pequenas e médias propriedades nas regiões estudadas.

\section{AGRADECIMENTOS}

Este estudo é fruto de um árduo trabalho de campo para a certificação florestal de origem da madeira em uma empresa da região. Gostaríamos de agradecer a todos os produtores florestais, empreiteiros e integrantes da cadeia produtiva de madeira que conhecemos e que compartilharam suas experiências, informações e expectativas para o setor, tendo contribuído com informações valiosas e atuais do estado da arte da cadeia produtiva de pequenos e médios produtores nas mesorregiões estudadas. Por fim, gostaríamos de agradecer ao trabalho impecável realizado por Angela Maria Appelt para a certificação realizada.

\section{REFERÊNCIAS}

CASTRO, A.W.V. et al. Cadeias produtivas do agronegócio florestal na região sul do Brasil. 2008. Disponível em: http://cdn.fee.tche.br/jornadas/2/E13-06.pdf

DAVID, H.C. et al. Economic analysis and revenue optimization in management regimes of Pinus taeda. Revista Ceres, v.64, n.3, p.222-231, 2017.

FISCHER, A.; ZYLBERSZTAJN, D. Fomento florestal como alternativa de suprimento de matéria-prima na indústria brasileira de celulose. Revista Eletrônica de Administração, v.72, n.2, p.494-520, 2012.

JONES, P.D. et al. Financial analysis of intensive pine plantation establishment. Journal of Forest Economics, v.16, p.101-112, 2010.

KOHLER, S.V. et al. Evolução do sortimento em povoamentos de Pinus taeda nos estados do Paraná e Santa Catarina. Floresta, v.45, n.3, p.545-554, 2015.

MOREIRA, J.M.M.Á.P. et al. Importância e desempenho das florestas plantadas no contexto do agronegócio brasileiro. Floresta, v.47, n.1, p.85-94, 2017.

SILVA, M.L. et al. Economia florestal. 2.ed. Viçosa: UFV, 2005.

SIMIONI, F.J.; HOEFLICH, V.A. Análise prospectiva da cadeia produtiva de energia de biomassa na região do planalto sul de Santa Catarina: Sistemas agroalimentares e cadeias agroindustriais. 45 Congresso da SOBER, n.1, p.1-15, 2007.

VASQUES, A.G.et al. Uma síntese da contribuição do gênero Pinus para o desenvolvimento sustentável no Sul do Brasil. Floresta, v.37, n.3, p.445-450, 2007.

Recebido em 02-05-2019 Aceito em 07-06-2019 